\title{
PISADAS FÓSILES DE MAMÍFEROS EN EL MIOCENO SUPERIOR DE LA HOYA DE LA SIMA (JUMILLA, MURCIA. ESPAÑA)
}

\author{
Félix PÉREZ-LORENTE ${ }^{\prime, 2}$, Francisco SERRANO \\ Tomás RODRÍGUEZ4, Miguel Ángel MANCHEÑO $O^{4}$ y \\ Mariela ROMERO ${ }^{2}$
}

\author{
'Universidad de La Rioja. Obispo Bustamante, 3, E-26001 Logroño. \\ ${ }^{2}$ Centro Paleontológico. E-26586 Enciso, La Rioja. \\ ${ }^{3}$ Departamento de Geología, Universidad de Málaga. Campus de Teatinos s/n, E-29071 \\ Málaga. \\ "Departamento de Química Agrícola, Geología y Edafología, Universidad de Murcia. \\ Campus de Espinardo, E-30071 Espinardo (Murcia).
}

Pérez-Lorente, F., Serrano, F., Rodríguez, T., Mancheño, M.A. y Romero, M. 1999. Pisadas fósiles de mamíferos en el Mioceno Superior de la Hoya de la Sima (Jumilla, Murcia. España) [Upper Miocene mammal footprints at la Hoya de la Sima (Jumilla, Murcia. Spain).] Revista Española de Paleontología, 14(2), 257-267. ISSN 0213-6937.

\begin{abstract}
In an old quarry of the Jumilla-Ontur Tertiary basin, located in the province of Murcia, mammal footprints are found on upper surfaces of gypsum deposits. From their study we can distinguish: parallel trackways of Hippipeda and Megapecoripeda made by gregarious equids and artiodactyls; a Carnivoripeda trail and an isolated footprint of Proboscipeda. The microfossils found immediately below the gypsum deposits are marine and represent the lowest Upper Tortonian. The change from marine to continental sedimentary conditions could be related to the Messinian crisis.
\end{abstract}

Keywords: Footprints, Mammals, Upper Miocene, Murcia.

\section{RESUMEN}

En la cuenca terciaria de Jumilla-Ontur (Murcia) se han encontrado huellas de mamífero fosilizadas en depósitos yesíferos aflorantes en una cantera antigua. El estudio de las mismas ha permitido distinguir huellas de Hippipeda y Megapecoripeda dejadas por grupos de équidos y de pecorinos, de comportamiento gregario, además de pisadas aisladas de Carnivoripeda y de Proboscipeda. La microfauna encontrada en los niveles infrayacentes es de la base del Tortoniense superior, y de carácter marino. El paso de condiciones marinas a continentales en la zona pudo estar relacionado con la Crisis messiniense.

Palabras clave: Pisadas fósiles, Mamíferos, Mioceno Superior, Murcia.

\section{INTRODUCCIÓN}

En este trabajo se describen varias pisadas fósiles de mamíferos encontradas por Cayetano Herrero (Herrero, 1997) en el paraje conocido con el nombre de la Hoya de la Sima. Aunque su estado de conservación es bueno, no lo son tanto sus detalles, por lo que su identificación no es muy estricta, y no se puede descender por debajo de la categoría de icnogénero. En España, hasta ahora, sólamente se habían citado pisadas de vertebrados del Mioceno en La Rioja (López et al., 1992) y en Álava (Antón et al., 1993), pero la edad de tales yacimientos es Mioceno Inferior (cf. Barot et al., 1996), mientras que las que se describen aquí pertenecen al Mioceno Superior, tratándose por tanto del único yacimiento español de huellas de vertebrados de esta edad. Las icnitas fósiles de mamíferos más próximas se encuentran en la provincia de Alicante (Sierra del Colmenar), pero son posteriores, Plioceno, y corresponden a pisadas de équidos y úrsidos (Lancis y Estévez, 1992).

Para la nomenclatura de las huellas se ha seguido la de Vialov (1966), dado que hay investigadores que afirman que es el sistema más apropiado para huellas de aves y mamíferos, que utiliza nombres de género y especie (Scrivner y Bottjer, 1986).

El yacimiento se encuentra en una antigua cantera de yeso, situada dentro del término municipal de Jumilla, junto a un camino que se toma entre los $\mathrm{Km} 8$ y 9 de la 

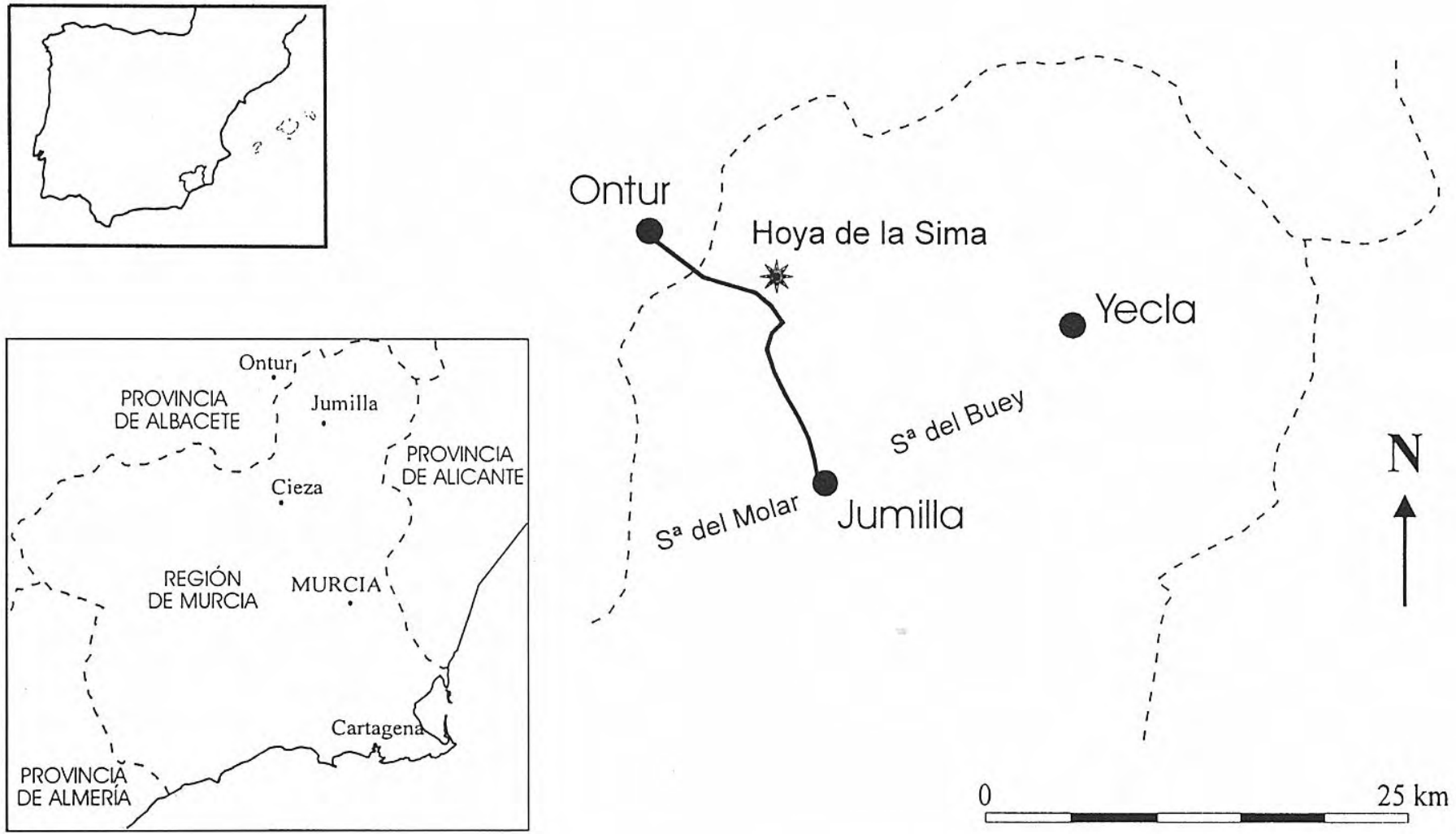

Figura 1. Localización del yacimiento.

carretera comarcal MU-403, a 13,3 Km de Jumilla (Fig. 1). Las coordenadas UTM del punto, referidas al mapa 1:50.000 (Hoja n 844 ONTUR) son 30SXH388702. De esta cantera se extrajeron también bloques aplanados rectangulares que sirvieron para enlosar varios edificios. Algunos de los bloques, que también contienen huellas, están depositados en el Museo Municipal y en el recinto del castillo de Jumilla.

Desde el punto de vista geológico, el sustrato de los materiales terciarios del yacimiento pertenece al Prebético interno, aunque esta localidad está muy cerca de afloramientos del Prebético externo. Los yesos ocupan una superficie de unos $42.000 \mathrm{~m}^{2}$, en la mitad meridional de la cuenca neógena marino-continental de JumillaOntur. En el centro de esta cuenca hay un diapiro de rocas triásicas que limita por el norte la mancha de yesos.

\section{ESTRATIGRAFÍA}

Se han levantado tres series estratigráficas (Fig. 2) con el fin de situar los niveles de yesos mencionados. A continuación se describen estas series cuyas secuencias se dan de muro a techo. Los números asignados a cada tramo son los de la secuencia ideal más completa que se deduce de la correlación de todas las series. En algunas de ellas faltan tramos inferiores, intermedios o superiores, debido a erosión (a partir del tramo 3 en la primera serie, del tramo 3 al 8 en la segunda y el tramo 8 de la tercera) o a que no aflora en el terreno (tramos 1 y 2 de las series segunda y tercera).
Serie de la Casilla del Yeclano. En el contacto con el diapiro de Ontur.

Tramo 1.- $10 \mathrm{~m}$ de conglomerados poligénicos.

Tramo 2.- $5 \mathrm{~m}$ de areniscas claras, poco cementadas, en bancos inferiores a $25 \mathrm{~cm}$ de espesor.

Tramo 3.- Margas blancas en las que hay intercalaciones de calizas blancas arenosas con cemento micrítico y con estructura subnodulosa. En la parte central de la cuenca, bajo los yesos, llega a tener 100 metros de potencia, deducidos a partir de perfiles geofísicos.

Serie de las Casas del Portal. Situada un $\mathrm{Km}$ al sur de la anterior, sería la continuación de la serie ya descrita.

Tramo 3.- Más de $15 \mathrm{~m}$ de margas blancas con intercalaciones de calizas blancas arenosas subnodulosas.

Tramo 8.- $3 \mathrm{~m}$ de areniscas, microconglomerados y margas, de color salmón, que reposan sobre el tramo anterior en aparente concordancia. Aunque no hay fósiles, se le atribuye una edad Plioceno en el mapa 1:50.000 del Plan Magna (García et al., 1984).

Tramo 9.- $1 \mathrm{~m}$ de conglomerados de colores claros, que incluyen costras calcáreas, discordante sobre el tramo anterior. Se atribuye al Cuaternario en la misma fuente que la citada en el tramo 8.

Serie de la Hoya de la Sima. Levantada en el extremo oriental del afloramiento de yesos de la cantera. El tramo inferior se encuentra discordante sobre unas calcarenitas del Mioceno Medio. En ella se distinguen los siguientes materiales:

Tramo 3.- $40 \mathrm{~m}$ de areniscas blancas correlacionables con el tramo 3 de las series anteriores. 


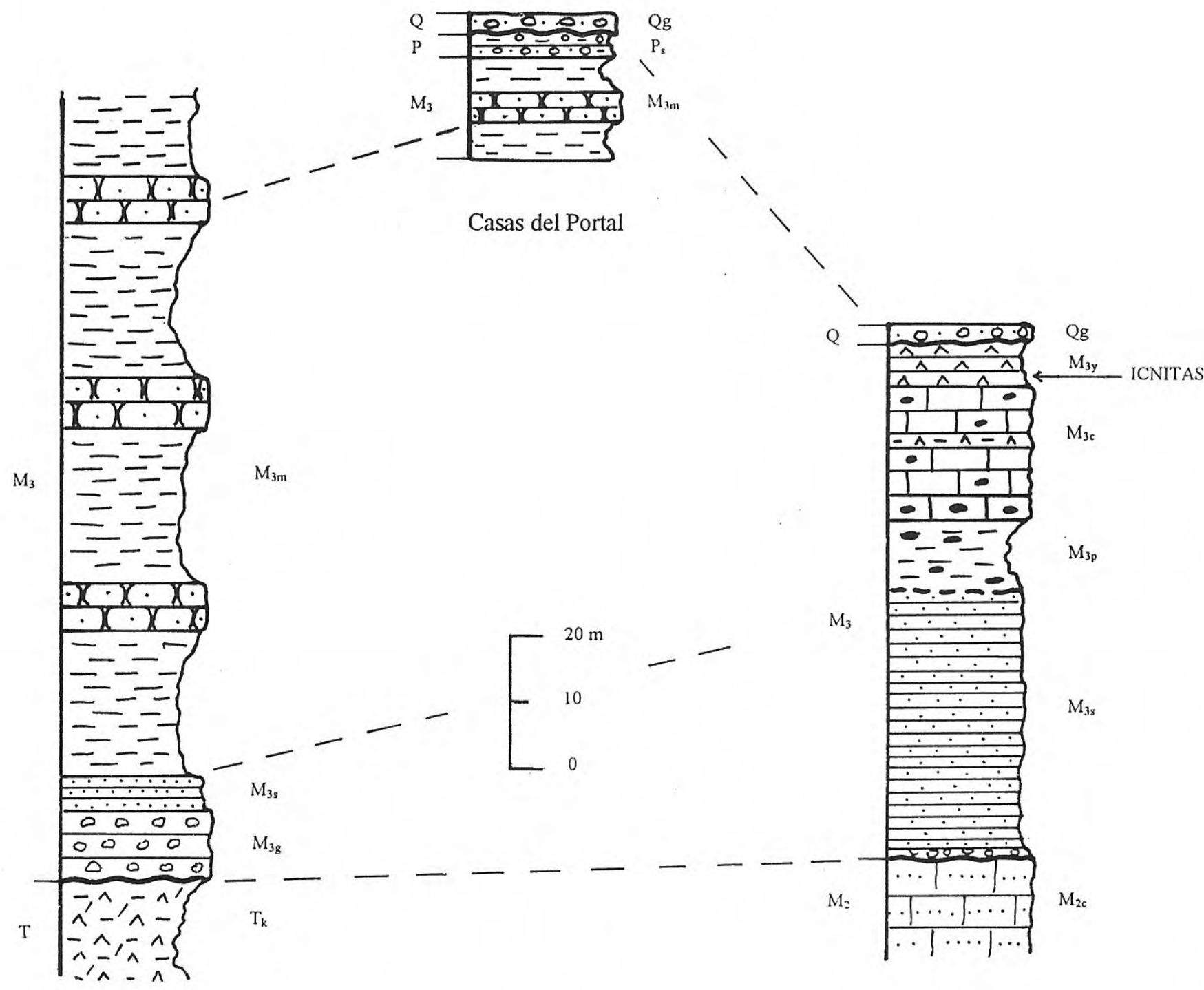

Casilla del Yeclano

Hoya de la Sima

Figura 2. Columnas estratigráficas. Leyenda: T: Triásico; $\mathrm{M}_{2}$ : Mioceno Medio; $\mathrm{M}_{3}$ : Mioceno Superior; P: Plioceno; Q: Cuaternario. $\mathrm{T}_{\mathrm{k}}$ : margas abigarradas con yeso; $\mathrm{M}_{2 \mathrm{c}}$ : calcarenitas; $\mathrm{M}_{3 \mathrm{~g}}$ : conglomerados; $\mathrm{M}_{3 \mathrm{~s}}$ : areniscas; $\mathrm{M}_{3 \mathrm{~m}}$ : margas con bancos de caliza micrítica arenosa subnodulosa; $\mathrm{M}_{3 \mathrm{p}}$ : margas bituminosas con sílex; $\mathrm{M}_{3 \mathrm{c}}$ : Calizas con sílex e intercalaciones de lechos de yeso; $\mathrm{M}_{3 y}$ : yesos en bancos potentes - hacia la base icnitas de vertebrados; $\mathrm{Q}_{\mathrm{g}}$ : conglomerados de matriz arcillosa y niveles encostrados.

Tramo 4.- $10 \mathrm{~m}$ de margas bituminosas oscuras con estructura laminada fina de tipo "paper shale" y sílex noduloso de color oscuro.

Tramo 5.- $3 \mathrm{~m}$ de calizas claras microcristalinas con abundante sílex.

Tramo 6.- $15 \mathrm{~m}$ de calizas blancas con sílex, margas y niveles de yesos rojos. En el techo hay calizas laminadas de algas.

Tramo 7.- $6 \mathrm{~m}$ de yesos blancos en bancos bien estratificados, cuya potencia oscila entre 6 y $60 \mathrm{~cm}$, que contienen las huellas de vertebrados. En la base de este tramo la estructura es diferente porque las capas están formadas por fragmentos de yesos anastomosados. En todo el tramo hay sílex de color blanco-grisáceo a acaramelado que se encuentra formando tanto nódulos como capitas cuyo espesor máximo es de $10 \mathrm{~cm}$.
Tramo 9.- $1 \mathrm{~m}$ de conglomerados con niveles de costras calcáreas del Cuaternario.

\section{DESCRIPCIÓN GEOLÓGICA DEL YACIMIENTO Y ALREDEDORES}

El yacimiento se encuentra en uno de los niveles inferiores de la parte bien estratificada del tramo 7, en el techo de un estrato de dirección N55E que buza entre 5 y $7^{\circ}$ hacia el Norte. No es un tramo de gran continuidad lateral, y parece que se encuentra sólamente en el centro de esta cuenca (esta afirmación está confirmada por campañas de geofísica realizadas por uno de nosotros, T. Rodríguez). No hay cambio de facies lateral, y el contacto inferior sobre las formaciones marinas es brusco y neto. 


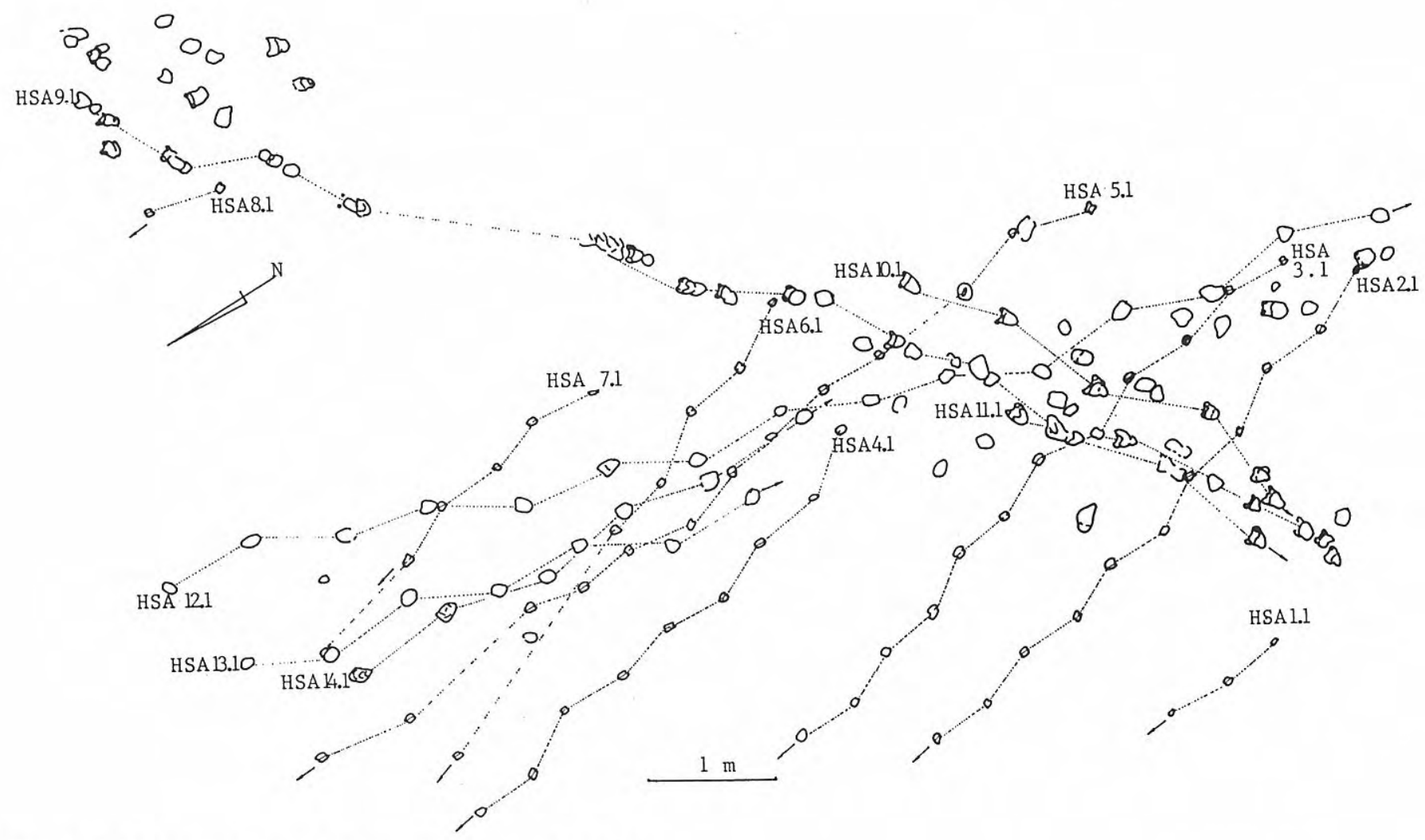

Figura 3. Cartografía de las pisadas del yacimiento HSA.

En general, el buzamiento de las capas miocenas es suave (inferior a $5^{\circ}$ ) excepto en el contacto con el diapiro de rocas triásicas de Ontur, en donde el arrastre debido a la halocinesis hace que el buzamiento sea de hasta $75^{\circ}$. Hay otras variaciones del valor del buzamiento, debidas a subsidencia diferencial de un surco periférico asociado al diapiro de Ontur, que no influyen en la disposición general presentada.

En conjunto, en el tramo 7 (yesos) de la Hoya de la Sima hay un sinclinal irregular, de dirección NE-SO, limitado al Norte por el diapiro de Ontur. En el afloramiento se observan también fracturas pequeñas y diaclasas, cuya dirección predominante es N-S.

Sobre el yeso se desarrolla una carstificación de poca relevancia, que se manifiesta por lapiaces y dolinas coalescentes que llegan a formar una dolina estructural de dirección coincidente con la del sinclinal mencionado debida a plegamiento neotectónico. En el fondo de algunas dolinas hay ponor-simas, lo que justifica el toponímico de "Hoya de la Sima" para este lugar.

\section{CONTENIDO FOSILÍFERO}

Con excepción de las pisadas de mamíferos, no se han encontrado otros restos fósiles en los yesos. Por el contrario, en los niveles margosos infrayacentes hay microfósiles, principalmente caparazones de foraminíferos bentónicos calcáreos, radiolas de equinodermos y, en algunas muestras, restos de peces. En menor proporción, también se detectan fósiles de ostrácodos y foraminíferos planctónicos. Esta microfauna pone de manifiesto que los niveles infrayesíferos se han depositado en un medio marino de plataforma abierta.

En las asociaciones de foraminíferos planctónicos se han identificado Globigerina bulloides (d'Orbigny), $G$. praebulloides (Blow), Orbulina universa (d'Orbigny), Neogloboquadrina continuosa (Blow), N. acostaensis (Blow), Globorotalia scitula (Grady), Globigirenita juvenilis (Bolli) y Turborotalita quinqueloba (Natland). La presencia de $N$. acostaensis indica que los sedimentos no son anteriores a la zona N.16 de Blow (1969), lo que condiciona una edad tortoniense o más moderna. La falta de ejemplares típicos de Neogloboquadrina humerosa (Takayanagi y Saito) podría indicar que los sedimentos están limitados al Tortoniense inferior, pero la escasez de componentes planctónicos minusvalora los criterios de ausencia. Un evento bioestratigráfico de mayor interés es que los ejemplares de Neogloboquadrina se presentan indistintamente con crecimiento dextrorso o sinistrorso. Este evento se ha detectado en las cuencas béticas y, en general, en el Mediterráneo occidental en dos momentos del Mioceno Superior:

a) En las proximidades del límite entre las zonas N.16 y N.17, (Blow, 1969), en relación con un cambio de dextrorso a sinistrorso en el enrollamiento predominante de Neogloboquadrina (Serrano et al., 1995). El cambio de enrollamiento es ligeramente posterior a la aparición de las primeras formas asimilables a $N$. humerosa (formas primitivas de tipo $N$. praehumerosa Natori). Si se utiliza la primera aparición de $N$. humerosa para marcar 


\section{$10 \mathrm{~cm}$}

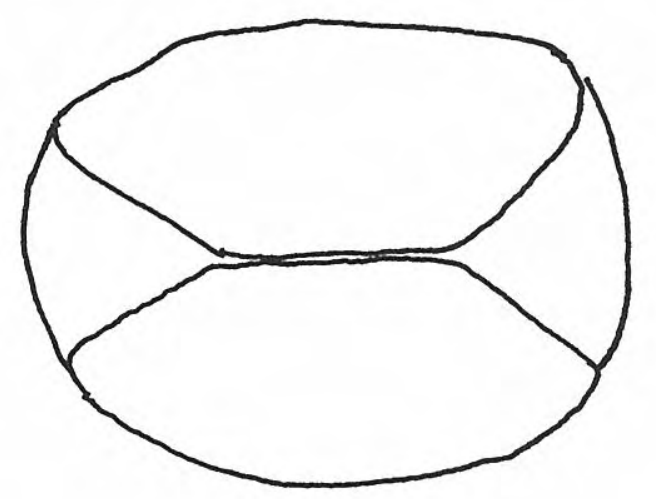

Figura 4. Dibujo a escala de una icnita procedente de la Hoya de la Sima. Museo de Jumilla.

el comienzo del Tortoniense superior (Serrano, 1979) este evento ocurriría al comienzo del Tortoniense superior.

b) En niveles relacionados con el comienzo de la crisis de salinidad messiniense, cuando se produce el cambio de sinistrorso a dextrorso en el sentido de enrollamiento de Neogloboquadrina. Este cambio que sigue a la primera aparición de G. elongatus (d'Orbigny) puede ser utilizado para marcar la base del Messiniense superior (Serrano, 1979, 1992). En este segundo caso, los niveles estudiados se habrían depositado al comienzo del Messiniense superior.

En principio, la presencia de yeso por encima de los niveles con microfauna, induce a correlacionarlos con los depósitos messinienses de las cuencas perimediterráneas. Sin embargo, la mayoría de los ejemplares de Neogloboquadrina corresponden a formas de $N$. continuosa, lo que parece más congruente con una edad Tortoniense. Por otra parte, no es rara la presencia de yeso en depósitos tortonienses de la Cordillera Bética (Dronkert et al., 1979). En el Sector central de dicha cordillera marcan el cierre de un ciclo sedimentario que termina con el régimen marino de las cuencas [v.g. Cuenca de Granada (González-Donoso, 1967; Dabrio et al., 1972; Rodríguez Fernández, 1982)]. En consecuencia, aunque ahora no se dispone de criterios definitivos, parece más probable que los yesos de la cuenca de Jumilla-Ontur y su secuencia marina infrayacente se depositaran hacia el comienzo del Tortoniense superior.

\section{PALEOAMBIENTE}

El contenido micropaleontológico mencionado en el apartado anterior pone de manifiesto que los niveles infrayesíferos (tramo 3 e inferiores) se han depositado en un medio marino de plataforma abierta en el Tortoniense superior. El tramo 4, con sus pizarras bituminosas, indica falta de oxígeno y la consecuente muerte masiva de organismos, lo cual es congruente con una regresión. Sobre este tramo, los depósitos de calizas, calizas algales

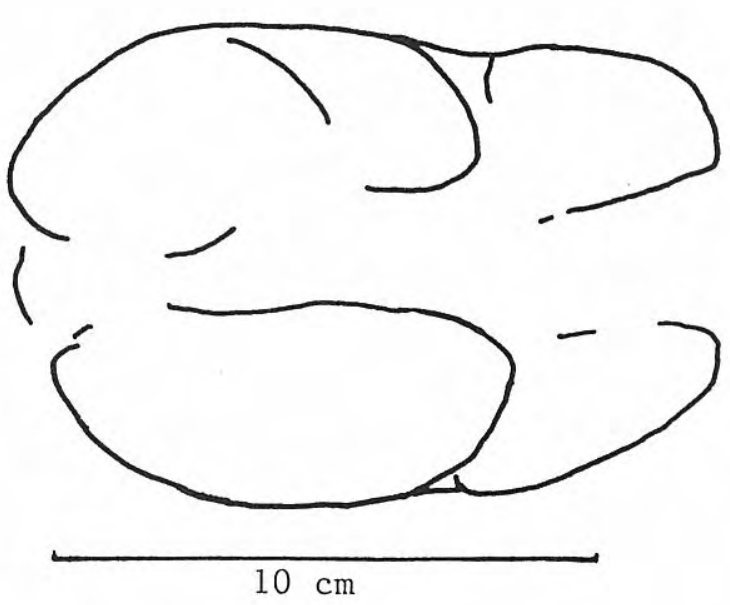

Figura 5. Dibujo a escala de HSM1.

y yesos (todos con sílex) indican ambiente continental lacustre cada vez más restringido en el que se produce aumento de la salinidad y de la concentración de magnesio.

Tras una etapa regresiva al comienzo del Tortoniense superior se instala, sólo en esta cuenca de Jumilla-Ontur, un lago que pierde relación con el ambiente marino. La formación de este lago vendría ocasionada por dos fenómenos tectónicos: uno la halocinesis del Trias diapírico de Ontur, que habría ocasionado un surco periférico secundario a su alrededor, una subsidencia diferencial y una depresión geomorfológica; y otro, el propio plegamiento mioceno de la zona, que creó un sinclinal aprovechado por el lago, y un anticlinal inmediatamente al norte de él, ambos con dirección NESO.

La secuencia de capas con yeso, y el hecho de que haya huellas de mamíferos en varias de ellas, indica que se trata de una depresión interior en la que, en ocasiones, se acumulaba agua en un lago que tendría periodos de sequía total. Muy probablemente, debido al tipo de animales que vivían en su entorno, el agua del lago sería dulce durante una parte de su permanencia.

\section{ESTUDIO ICNÍTICO}

La Hoya de la Sima suministra huellas (huecos y rellenos) de mamíferos terciarios. Existen actualmente dos sectores separados por 30 metros en los que afloran pisadas de mamíferos grandes en una cantera antigua situada en el paraje citado. También se encuentran improntas estampadas en bloques extraídos para solería, hoy en salas del Museo Municipal y en el recinto del castillo de Jumilla.

A los dos sectores los llamaremos Hoya de la Sima A y Hoya de la Sima B, el segundo al suroeste del primero. En los dos se reconocen grupos de pisadas. El número de rastros es mayor en el primero (A) que en el segundo (B), más pequeño. Cada icnita se designa con una secuencia de guarismos y dígitos, como en los ejemplos que se explican, HSA4.3 o HSA52, y cuyo significado es: 


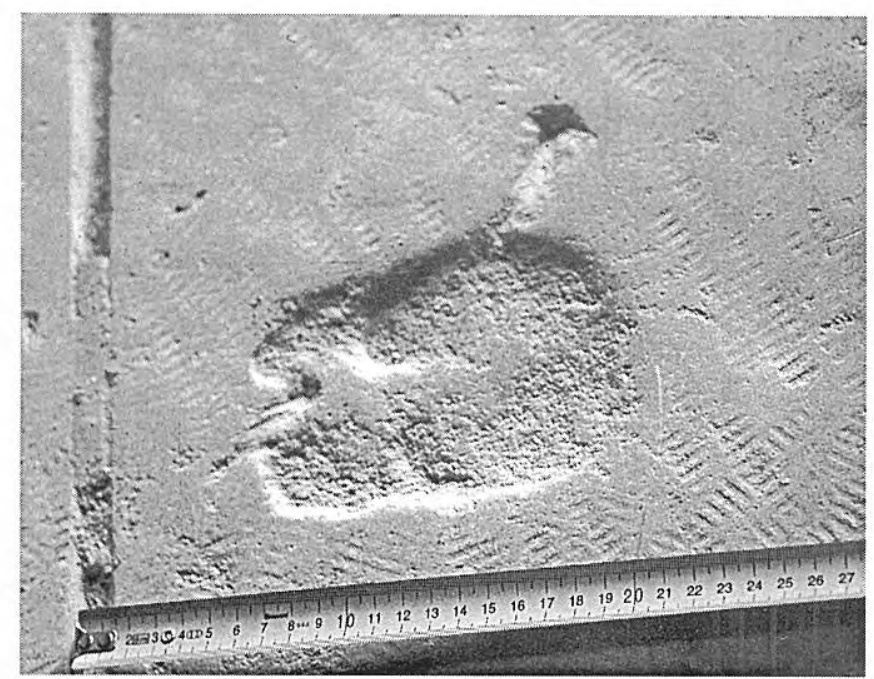

Figura 6. Fotografía de la icnita HSM1.

HS: yacimiento de la Hoya de la Sima

A: sector A del yacimiento

4 o 52: asociación de pisadas número 4 o huella aislada número 52

4.3: pisada tercera de la rastrillada 4.

Aunque en algunos casos no se conoce el sentido de marcha, se ha adoptado el que se indica en la Fig. 3, de manera que la primera huella impresa de cada pista tiene el número 1. Si falta alguna icnita se mantiene el orden y la secuencia en la nomenclatura de las pisadas restantes, como si estuviesen impresas absolutamente todas las del rastro. De esta manera, contabilizamos las rastrilladas HSA1 hasta la HSA11 (ambas incluidas) y las icnitas aisladas HSA12 hasta la HSA65, ambas incluidas.

\section{AFLORAMIENTO HSA (Fig. 3)}

Su extensión es de unos $45 \mathrm{~m}^{2}$. En él se encuentran varios rastros muy claros, asociados en tres grupos.

- Siete rastrilladas paralelas que contienen 60 icnitas.

- Un rastro cuadrúpedo con 22 icnitas (11 de manos y 11 de pies).

- Tres rastros con 29 icnitas.

El número total de huellas reconocidas, las de los rastros mencionados y las icnitas aisladas no asociables a secuencia alguna, asciende a 163.

\section{Rastrilladas HSA1, HSA2, HSA3, HSA4, HSA5, HSA6, HSA7, HSA8}

Cada pisada deja dos impresiones muy poco profundas en las que se aprecia la separación entre dos dedos (Fig. 4). El septo interdigital que separa los dos lóbulos es muy estrecho y a veces no se ve. La profundidad de las dos señales es similar en toda su extensión. No hay disimetría ni entre los dos lóbulos ni en un mismo lóbulo, tanto sagital (las dos pezuñas son iguales y están igualmente dispuestas) como transversalmente (no terminan en punta, ni son más estrechas por un lado que por otro), de manera que no se puede diferenciar ni parte anterior ni posterior del conjunto. Las terminaciones anteriores y posteriores de los lóbulos y del pie en su conjunto, son redondeadas. No

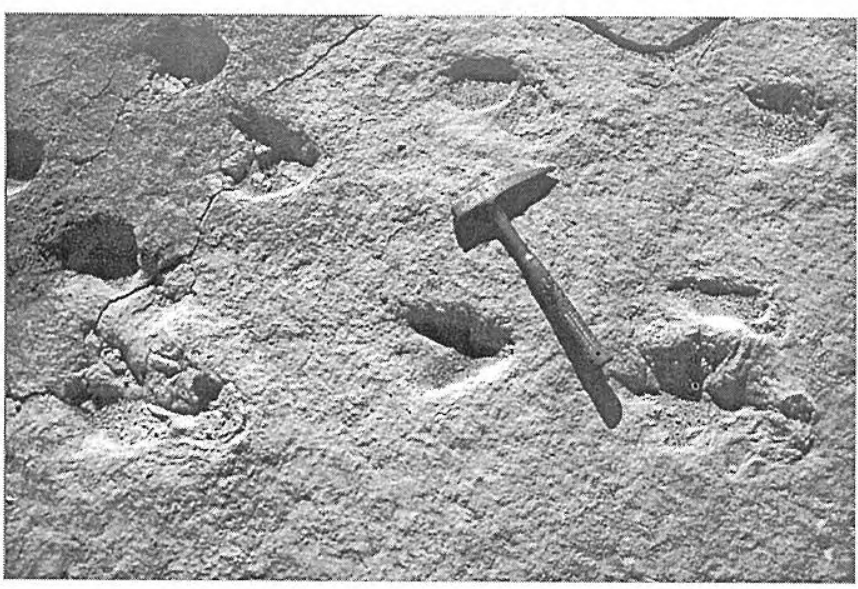

Figura 7. Fotografía de marcas de équidos. Detalle del yacimiento HSA.

se observa divergencia entre las extremidades de las pezuñas. Estas quizá tengan envuelta exterior, ya que también hay impresión sobre el suelo en la incisión que se encuentra en el inicio y terminación de las pezuñas. Casi todas las rastrilladas están completas; es decir, no faltan icnitas entre la primera y la última de la secuencia de improntas que las definen, a excepción de HSA5.4, HSA 5.13, HSA5.14, HSA6.6, HSA6.7, HSA6.8 y HSA7.7.

Para conocer el sentido de marcha se ha adoptado la parte positiva (sensu Leonardi, 1987) de la orientación del eje del pie con respecto a la línea media del rastro. Según esto, se deduce que iban hacia el Norte, como se indica en la Fig. 3, por la secuencia de pisadas de cada pista (la 1 es la primera que se marca).

En la Tabla 1 están los valores medios encontrados para estos rastros. Se observa la poca variación de las cifras y la correspondencia entre el valor grande y pequeño de I (longitud de la pisada) con el grande y pequeño de a (anchura de la pisada). La rastrillada HSA1, que proporciona un valor medio de $65 \mathrm{~mm}$ para la longitud del pie, no es fiable porque sólamente tiene tres icnitas. La relación entre la longitud del pie y su anchura es relativamente baja; $(\mathbf{I}-\mathbf{a}) / \mathbf{a}$ oscila entre $-0,080$ y 0,246 .

Son marcas típicas de artiodáctilos de pezuñas paralelas. Las que se observan parecen ser sólamente las dejadas por el pie; no se ve ninguna señal de las manos. En este tipo de rastros es normal que no haya huellas de las manos. Se pueden barajar al menos tres hipótesis para que este hecho ocurra:

- Que la pisada de la mano, probablemente más larga que la del pie, produzca cierta perturbación en el suelo pero que no se hunda en él debido a las características del barro, y que después el pie penetre en el fango aprovechando la perturbación previa y así quede marcado (Ellemberger, 1980).

- Que se produzca la superposición total de la pisada del pie sobre la de la mano (cf. Bang y Dahlström, 1997).

- Que el tipo de marcha produzca gran separación entre las huellas de la mano y la del pie, como en el trote gorrinero ("running walk" cf. Renders, 1984). 


\begin{tabular}{|l|l|l|l|l|l|l|l|l|l|l|l|l|l|}
\hline Rastros & 1 & $\mathrm{a}$ & $\mathrm{P}$ & $\mathrm{z}$ & $\mathrm{Ar}$ & $\mathrm{Lr}$ & $\mathrm{Ap}$ & $\mathrm{O}$ & Long & $\mathrm{Ar} / \mathrm{a}$ & $\mathrm{g}-\mathrm{a}$ & Direc & $\mathrm{N}^{\circ}$ hll \\
\hline HSA1 & 6,5 & 6,4 & 48,5 & 96 & 2 & 10,5 & 170 & 10 & 102 & 0,31 & 75 & N357E & 3 \\
\hline HSA2 & 8,3 & 6,8 & 49 & 96 & 5 & 17 & 152 & 10 & 490 & 0,73 & 71 & N343E & 11 \\
\hline HSA3 & 8,4 & 7,1 & 48 & 94 & 5 & 18,5 & 154 & 7 & 525 & 0,70 & 72 & N346E & 12 \\
\hline HSA4 & 8,1 & 6,6 & 51 & 100 & 5 & 17 & 156 & 10 & 407 & 0,76 & 76 & N349E & 9 \\
\hline HSA5 & 8,9 & 7,2 & 47,5 & 94 & 4 & 14 & 162 & 8 & 530 & 0,55 & 74 & N338E & 10 \\
\hline HSA6 & 8 & 6,5 & 53 & 105 & 5 & 17 & 163 & 5 & 425 & 0,76 & 79 & N356E & 7 \\
\hline HSA7 & 8,6 & 6,9 & 50 & 95 & 5 & 16 & 156 & 4 & 294 & 0,72 & 70 & N347E & 6 \\
\hline HSA8 & 8 & 8,7 & 57 & - & - & - & - & - & 65 & - & - & - & 2 \\
\hline HSA9 & 14,5 & 11 & 70,8 & 137 & 7 & 25,1 & 157 & - & 950 & 0,64 & 102 & N200E & 15 \\
\hline HSA10 & 12,9 & 10,8 & 69,8 & 137 & 9,2 & 29,2 & 150 & - & 550 & 0,72 & 102 & N194E & 8 \\
\hline HSA11 & 15,1 & 12,3 & 78,7 & 152 & 6,2 & 24,7 & 161 & - & 406 & 0,41 & 114 & N184E & 6 \\
\hline HSA12 p & 14,5 & 14,0 & 75,4 & 149 & 7,5 & 29,2 & 155 & - & 960 & 0,52 & 125 & N227E & 21 \\
HSA12 m & 9,3 & 11,6 & 76,8 & 144,7 & 7,6 & 26,4 & 153 & - & & 0,65 & & N240E & \\
\hline HSA13 & 14,9 & 13,7 & 82,8 & 162,9 & 9,6 & 34,2 & 153 & - & 330 & 0,70 & 121 & N240E & 5 \\
\hline HSA14 & 14,4 & 12,6 & 77,1 & 154,4 & 2,1 & 20 & 174 & - & 250 & 0,17 & 112 & N232E & 4 \\
\hline
\end{tabular}

1 - longitud de la huella; a - anchura; P - longitud del paso; z - longitud de la zancada; Ar - amplitud de rastrillada; $\mathrm{Lr}$ luz de rastrillada; Ap - ángulo de paso; O - orientación; Long - longitud de la rastrillada; g-a - distancia glenoacetabular; Direc - dirección de la rastrillada y sentido de marcha; $\mathrm{N}^{\circ}$ hll - número de huellas de la rastrillada. Medidas en centímetros y en grados.

Tabla 1. Datos medios de las medidas tomadas en las icnitas de los catorce rastros del yacimiento de la Hoya de la Sima.

Si las marcas de manos y pies son similares, es posible que el animal vaya al galope, y que dos huellas consecutivas (izquierda y derecha o viceversa) sean las dejadas por el pie y las dos siguientes por la mano (página 58 de Bang y Dahlström, 1997).

No creemos que se trate del primer caso porque donde se ha citado, una vez rota la parte superior del barro, las extremidades posteriores penetran mucho (hasta $10 \mathrm{~cm}$; Ellemberger, 1980) dejando huellas muy profundas. En nuestro caso, la parte perturbada de barro y atravesada sería menor de un centímetro de profundidad.

Con respecto a la tercera posibilidad, en las marcas se detecta bien la separación entre las pezuñas, y sin embargo, ni hay indicación de que resbalara al trotar, ni la disposición de las manos y pies se corresponde con tal tipo de marcha. Las icnitas las dejaron animales que apoyaban sus extremidades de manera que quedaba el contorno bien señalado -sin alargamiento, al contrario de las descritas por Astibia et al. (1994)-, sin estrías en el fondo ni acumulación de barro delante o detrás de la huella. Además de estos detalles, la amplitud de rastrillada (Ar o distancia del centro del pie a la línea media de la pista) es muy grande; la relación entre Ar y a indica que son rastros anchos (entre 0,55 y 0,76 si exceptuamos el valor de 0,31 encontrado para la pista HSA1; ver Bang y Dahlström, 1997; Casanovas et al., 1995), más apropiados de andar lento que de un animal al galope.

Podría ocurrir, aunque este detalle no lo hemos podido comprobar en el afloramiento, que los animales pisaran encima de una capita de barro muy fina (menor de $1 \mathrm{~cm}$ ), de manera que la atravesaran para dejar la suela sobre la capa de yeso, aunque quizá sólo una marca parcial de la mano. Probablemente la huella dejada por la mano, ahora sobrepisada, fuera del mismo tamaño que la del pie, que es la que ahora se ve.

Si consideramos que son pisadas de animales que van al paso y en los que la superposición del pie sobre la mano es completa, se puede continuar el análisis del rastro para deducir las dimensiones del animal. Suponemos que el tipo de marcha era caminando con paso alternante evolucionado (Demathieu, 1987). Buscamos la distancia entre las cinturas escapular y pelviana (g-a, Tabla 1), según el método propuesto por este autor en la referencia citada. Tal dimensión (g-a) oscila entre 70 y $79 \mathrm{~cm}$, lo cual implica que la alzada del animal sería del orden de un metro.

\begin{tabular}{|l|l|l|l|l|l|l|l|llll|llll|}
\hline Huella & 1 & $\mathrm{a}$ & $\mathrm{P}$ & $\mathrm{z}$ & $\mathrm{Ar}$ & $\mathrm{Lr}$ & $\mathrm{Ap}$ & II & III & IV & V(i) & II & III & IV & V(ii) \\
\hline 3 & 115 & 105 & & & & & & 50 & 47 & 46 & 33 & 28 & 26 & 32 & 30 \\
\hline 2 & 123 & 111 & 590 & 1210 & 65 & 235 & 159 & 54 & 56 & 62 & 36 & 34 & 31 & 28 & 18 \\
\hline 1 & & & 650 & & & & & & & & & & & & \\
\hline media & 119 & 108 & 620 & 1210 & 65 & 235 & 159 & 52 & 51 & 54 & 34 & 31 & 28 & 30 & 24 \\
\hline
\end{tabular}

II III IV V (i) longitud de los dedos; II III IV V (ii) anchura de los dedos. El resto de símbolos es igual que el usado en la Tabla 1. Medidas en milímetros y en grados.

Tabla 2. Datos medios de medidas tomadas en las icnitas de la rastrillada HSB5. 


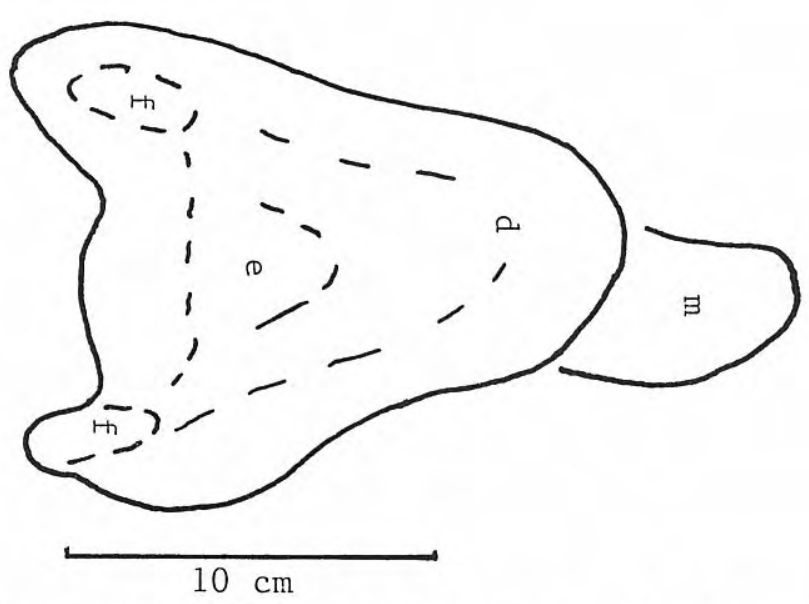

Figura 8. Dibujo a escala de HSM2. (m, d, e, f: explicación en el texto.)

En los bloques sueltos hay alguna marca que tiene más información que las encontradas en el campo. En la HSM1 (Figs. 5 y 6), depositada en el Museo Municipal de Jumilla, a pesar de que se trabajó su superficie para dejarla más lisa, hay una impronta en la que la superposición de la mano sobre el pie no es total (congruente con un animal sin grandes cornamentas ni desarrollo mayor de los cuartos delanteros que de los traseros). En ella, la marca de la mano no es más grande que la del pie; en cada señal de dedo de los pies se sugieren dos estructuras (una dejada por una almohadilla y otra por una pezuña o suela); la longitud de los dedos del pie es de $97 \mathrm{~mm}$ y su anchura $100 \mathrm{~mm}$, medidas similares a las de las rastrilladas HSA2 a HSA8.

Las características descritas son las del icnoorden Artiodactipedida (Vialov,1966), y por el tamaño, superior al de las icnitas representativas de Pecoripeda, se podrían incluir en el icnogénero Megapecoripeda de Kordos (1983).

En España se han descrito icnitas de este icnoorden en Lérida -Entelodontipus viai (Casanovas y Santafé, 1982) (también Prats y López, 1995) de edad Oligoceno medio-; en Navarra -Entelodontipus (Astibia et al., 1994) de edad Oligoceno inferior-; y, finalmente, en Álava -Artiodáctilo grande y Artiodáctilo pequeño (Antón et al., 1993) de edad Mioceno inferior.

\section{Rastrilladas HSA9, HSA10, HSA11 (Fig. 3)}

Son tres secuencias de marcas redondeadas, más profundas por una parte que por otra. En algunas se aprecia que son algo más abiertas por la parte menos profunda.

Entre estos tres rastros se observan algunas diferencias:

- HSA9 y HSA10 son paralelos, mientras que HSA11 los atraviesa.

- La magnitud de $\mathbf{I}, \mathbf{a}, \mathbf{P}$ (paso), z (zancada) y Ar/a es similar en HSA9 y HSA10 y distinta en HSA11; la amplitud de rastrillada (Ar) es mayor en HSA9 y HSA10 que en HSA11.

En la identificación de estas trazas hay que proceder por eliminación, ya que no hay señales suficientes que
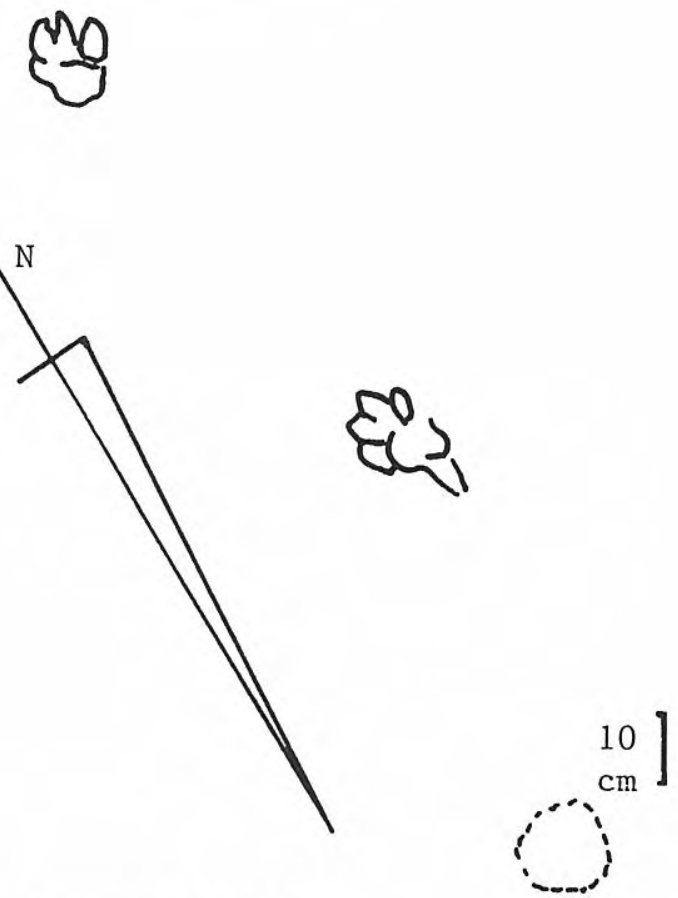

Figura 9. Rastrillada HSB5.

permitan encontrar caminos para realizarla. Debe tratarse de animales cuadrúpedos que superponen las pisadas de los pies a las de las manos, como en las huellas de las rastrilladas anteriores. El sentido de la marcha, deducido por la orientación positiva de las improntas es N200E para HSA9, N194E para HSA10, y N184E para HSA11. Para la identificación de los animales que las produjeron véanse las consideraciones hechas en la descripción de las rastrilladas siguientes.

\section{Rastrillada HSA12, HSA13, HSA14 (Fig. 3)}

Son rastrilladas en las que se encuentran series separadas de las huellas de dos elementos que se repiten y superponen. Donde se ven las dos pisadas, la avanzada (mano) no muestra ningún carácter especial -es redondeada y más profunda en su parte anterior que en la posterior-. La marca retrasada (pie) tiene también su parte delantera más profunda; hacia atrás, se levanta suavemente hasta llegar a un escalón en cuya meseta superior quedan señalados dos huecos laterales. Muchas veces la impresión delantera del pie tiene forma de uve abierta hacia atrás.

Las marcas son más anchas (en la parte posterior) que largas. Se ha hecho la media con 19 pisadas y el resultado es de $135 \times 125 \mathrm{~mm}$. Los dos huecos laterales son subcirculares u ovalados con el eje mayor coincidente con el del pie, y su diámetro medio es de $41 \mathrm{~mm}$. La profundidad máxima media de las 19 pisadas citadas es de $36 \mathrm{~mm}$. Estos caracteres permiten identificar las huellas como las dejadas por équidos que imprimían el dedo central (III) y dos dedos laterales más cortos (II y IV) (Fig. 7).

En el estudio de estas pisadas se encuentran dos casos: a) uno en el que la superposición de mano y pie es casi total; y b) otro en el que las marcas de la mano (m, Fig. 


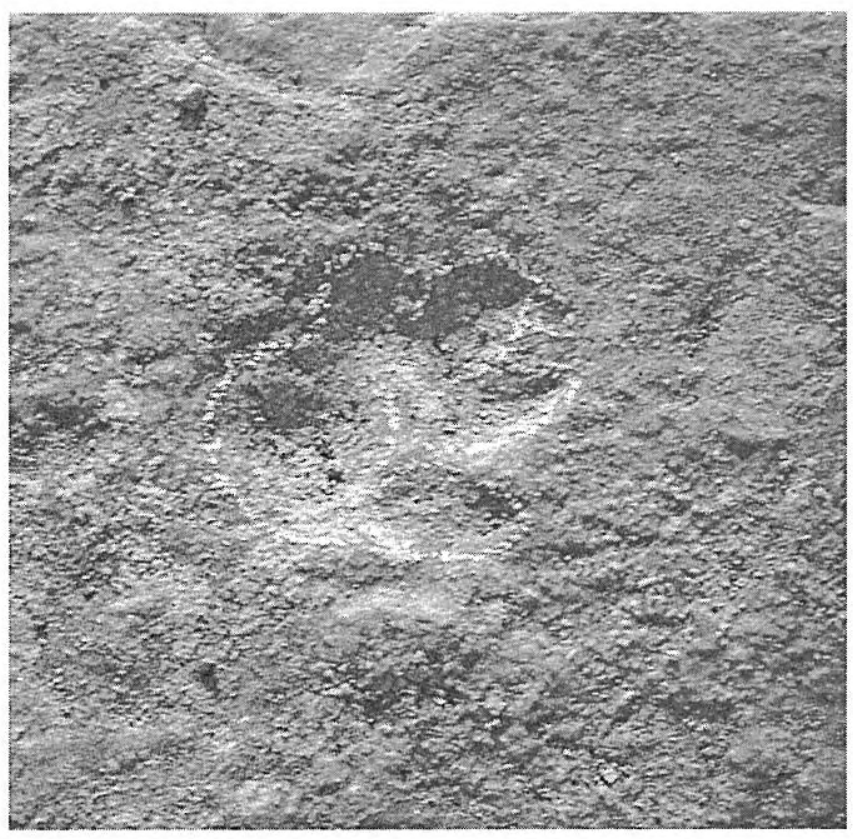

Figura 10. Fotografía de la icnita HSB5.3. Longitud de la huella $115 \mathrm{~mm}$.

8) y las del pie están bien separadas. En las huellas que están bien marcadas hay una parte anterior (d, Fig. 8), ancha y hundida, producida por la pezuña del dedo central (III); detrás de ella, una zona elevada (e, Fig. 8) que no llega al nivel del suelo; finalmente, en la parte más posterior, dos hendiduras (f, Fig. 8) alargadas o subredondeadas, que materializan los huecos dejados por los dos dedos II y IV. Estas huellas las atribuimos a Hipparion, perisodáctilo équido del Mioceno Superior. La distancia entre la cintura escapular y la pelviana de los animales que produjeron estas rastrilladas -calculada a partir de la hipótesis de que fueron al paso y con andar alternante evolucionado (Demathieu, 1987)- oscila entre $102 \mathrm{~cm}$ (rastrilladas HSA8, HSA9) y $125 \mathrm{~cm}$ (rastrillada HSA12).

En el conjunto del sector HSA hay tres series de icnitas que responderían a las mismas características biomórficas:

$1^{\text {a) }}$ Las rastrilladas HSA9, HSA10 y HSA11, cuyo sentido de marcha está entre N184E y N200E y en las que la superposición del pie sobre la mano es total.

$2^{a}$ ) Las rastrilladas HSA12, HSA13 y HSA14, cuyo sentido de marcha oscila entre N232E y N240E, en las que son abundantes las icnitas separadas de mano y pie.

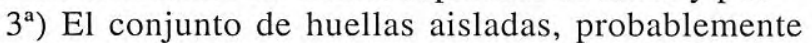
todas dejadas por el mismo tipo de animales (de HSA15 a HSA47).

\section{AFLORAMIENTO HSB}

Se han limpiado en él dos capas con huellas. La inferior contiene cuatro marcas sin ornamentación (de HSB1, HSB2, HSB3 a HSB4), similares a algunas de las de HSA9 a HSA14.

\section{Rastrillada HSB5 (Figs. 9 y 10)}

En la capa superior se ha encontrado una secuencia de

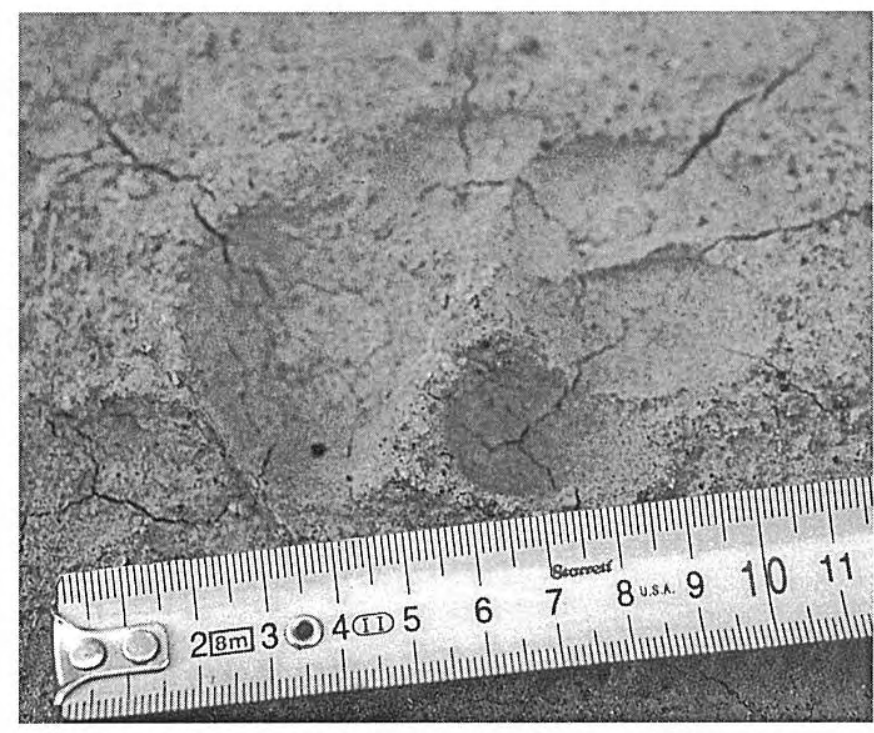

Figura 11. Fotografía de una huella actual de perro, sin señal de las uñas.

tres icnitas, cuyas características están en la Tabla 2. De estas marcas:

- HSB5.1 es redondeada;

- en HSB5.2 se distinguen bien los dedos pero la almohadilla plantar y el talón no son claros (en la parte posterior hay una señal posíblemente dejada al pisar);

- en HSB5.3 se distinguen muy bien los dedos puntiagudos y la almohadilla plantar.

Se reconocen cuatro dedos, los dos centrales igualmente adelantados. El dedo más pequeño de las huellas parece que es el externo. Se ha calculado la media de la longitud y anchura de los dedos en las dos huellas en las que se pueden medir; el resultado está en la Tabla 2.

Se trata, sin duda, de la pista dejada por un mamífero carnívoro que al andar sobrepone totalmente la pisada del pie a la de la mano. No es fácil distinguir qué tipo de pisada de carnívoro es, si de félido o de cánido (Panin y Avram, 1962), ya que, aunque la terminación de las marcas de los dedos en HSB5.3 (Fig. 10) es redondeada, esto no es un criterio definitivo de pisadas de félidos, pues en las pisadas de perros actuales a veces tampoco queda la impresión de las uñas (Fig. 11).

No se puede comparar con el resto de las pisadas de carnívoros encontradas hasta ahora en el Mioceno peninsular. Con respecto a las citadas por Antón et al. (1993) presentan:

1- Los dedos más unidos entre ellos y con la almohadilla plantar.

2- La longitud y anchura es más del doble (119x1908 $\mathrm{mm}$ en la Hoya de la Sima).

3- Los dedos exteriores (II y V) no están tan redondeados.

Con respecto a Carnotipus (López et al., 1992), tienen también caracteres diferentes, como son:

1- Carnotipus tiene los dedos separados tanto entre ellos como de la almohadilla plantar.

2- Carnotipus tiene los dedos más alargados y puntiagudos. 


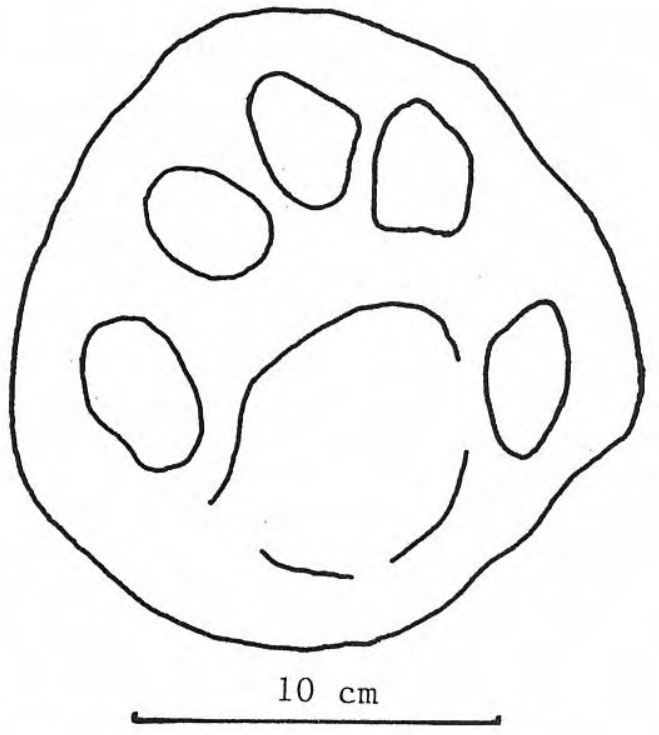

Figura 12. Dibujo a escala de HSM3.

\section{ICNITA HSM3 (Fig. 12)}

En uno de los bloques depositados en el Museo, hay una pisada constituída por una almohadilla plantar rodeada en su parte anterior por cinco huecos, de terminación redondeada, dejados por los dedos. Las dimensiones de la pisada son de $19,7 \times 18,3 \mathrm{~cm}$. La parte más profunda está hacia el centro de la almohadilla plantar. Es posible que haya cierta simetría en la disposición de los dedos relativa a un eje que pasa por el centro de la huella y a lo largo del dedo central (III). Teniendo en cuenta el número, forma (Panin y Avram,1962) y disposición de los dedos, y el tamaño de la marca, atribuimos esta señal a la dejada por un autopodio de un proboscídeo.

\section{CONSIDERACIONES PALEOETOLÓGICAS}

En este yacimiento se encuentran asociadas varias pistas y huellas aisladas, de al menos tres grupos de animales, un grupo de artiodáctilos y dos de équidos:

- Megapecoripeda, de artiodáctilos, de las que han quedado impresas 8 rastrilladas relativamente bien marcadas, paralelas en dirección y de igual sentido de marcha, separadas entre $30 \mathrm{~cm}$ y 1,2 metros, que atraviesan todo el afloramiento, recorriendo sobre él una distancia del orden de 5 metros. Parecen componentes de un grupo, no sabemos si muy numeroso, porque el afloramiento queda tapado en sus extremos, que andaban de paso y no se encontraban pastando o establecidos en un lugar. Por la distancia entre los rastros con relación al tamaño de los animales (de sus pisadas), parece más probable que se tratara de un grupo poco numeroso, ya que no se ven rastros superpuestos. Al menos hay tres bloques con icnitas de este tipo depositados en el Museo de Jumilla.
- Hippipeda, de Hipparion, de las que se han separado 6 rastros y unas 30 icnitas aisladas. No hay un solo sentido de marcha como en las pistas anteriores. Es probable que en este caso los animales se encontraran en el borde del lago, entre otras cosas porque la profundidad de sus huellas es mayor que en el caso anterior y por lo tanto el barro sería más blando. A las icnitas de Megapecoripeda HSA2.4, HSA3.2, HSA7.4 se superponen las HSA12.13m, HSA9.13, HSA9.4. Pensamos que se trata de un grupo de équidos que deambulaban por el lugar, de los que al menos hay rastros de 6 animales. Hay un número no determinado de bloques con pisadas de équidos en el Museo de Jumilla.

\section{CONCLUSIONES}

Se han encontrado nuevos afloramientos con huellas fósiles de mamíferos del Terciario. Estas huellas de mamíferos terrestres en sedimentos continentales, que se colocan directamente encima de otros con foraminíferos marinos de edad Tortoniense superior, hacen suponer que la edad del yacimiento es Tortoniense o bien Messiniense, coincidiendo en este caso, en el tiempo, con la llamada Crisis messiniense.

En este yacimiento se encuentran pisadas de Hipparion, de un pecorino, de un carnívoro y, probablemente, de un proboscídeo. Las de pecorino y las de équido se encuentran asociadas como si el comportamiento de estos dos tipos de animales fuera gregario. De los otros dos animales sólo se encuentran un rastro y huellas aisladas.

En este trabajo se describen por primera vez pisadas de Hipparion en España.

\section{AGRADECIMIENTOS}

Nuestro primer agradecimiento es para D. Emiliano Hernández Carrión y D. Cayetano Herrero González, director y subdirector de Museo de Jumilla, que nos comunicaron y condujeron al yacimiento. Gracias a sus gestiones, el Ayuntamiento de dicha localidad sufragó una parte importante de los gastos que se hicieron para hacer la cartografía y reproducción a escala del afloramiento. Finalmente, queremos agradecer a los Dres. Casanovas, investigadora del Instituto de Paleontología "M. Crusafont" de Sabadell, H. Astibia y E. Mayoral la corrección y sugerencias al trabajo.

\section{BIBLIOGRAFÍA}

Antón, M., López, G. y Santamaría, R. 1993. Estudio preliminar de la icnofauna miocena del yacimiento de Salinas de Araña (Provincia de Álava). Comunicaciones de las IX Jornadas de Paleontología, Málaga, 23-28.

Astibia, H., Del Valle, J. y Murelaga, X. 1994. Icnitas de artiodáctilos (Mammalia) del Paleógeno de Olcoz 
(Depresión del Ebro, Navarra). Estudios Geológicos, 50, 119-126.

Bang, P. y Dahlström, P. 1997. Huellas y señales de los animales de Europa. Omega, 1-243.

Barot, G., Soler, M., Aguilar, M., Fernández, J. y Quintana, J. 1996. Recopilación de las icnitas de mamíferos de la Península Ibérica. Universidad Autónoma de Barcelona. Memoria inédita, 1-27.

Blow, W.H. 1969. Late Middle Eocene to recent planktonic foraminiferal biostratigraphy. Proccedings First International Conference On Planktonic Microfossils, Ginebra, 1, 199-422.

Casanovas, M.L. y Santafé, J.V. 1982. Icnofauna oligocena de Agramunt (Lérida, España). Acta Geológica Hispánica, 17, 113-119.

Casanovas, M.L., Ezquerra, R., Fernández, A., PérezLorente, F., Santafé, J.V. y Torcida, F. 1995. Huellas de dinosaurio en la Era del Peladillo 3 (La Rioja. España). Primera nota. Zubía, 13, 83-101.

Chaline, J. 1990. Paleontology of vertebrates. Springer Verlag, 1-186.

Dabrio, C., García-Yebra, R., González-Donoso, J.M. y Vera, J.A. 1972. Turbiditas asociadas a evaporitas (Mioceno, La Malá, Depresión de Granada). Cuadernos de Geología, 3, 139-164.

Demathieu, G.R. 1987. Plate VIII. In: Glossary an manual of tetrapod footprint palaeonichnology (G. Leonardi ed.).

Dronkert, H., Poel, H. van der and Gerlings, L.P.A. 1979. Gypsum deposits in the province of Almería. Consequences for the western Mediterranean. Annales de Geologie des Pays Helleniques, 1, 345-354.

Ellemberger, P. 1980. Sur les empreintes de pas des gros mammiferes de l'Eocene superieur de Garrigues-SteEulalie (Gard). Palaeovertebrata, 14, 37-78.

García, A., López, F y Baena, J. 1984. Mapa Geológico de España. Escala 1:50.000. Hoja $n^{\circ} 844$ (ONTUR). IGME. Hoja y memoria.

González-Donoso, J.M. 1967. Estudio geológico de la Depresión de Granada. Tesis Doctoral Universidad de Granada, (inédita).

Herrero, C. 1997. Las huellas fósiles de la Hoya de la Sima. El Picach, 93, 21-22

Kordos, L., 1983. Lábnyomok az Ipolytarnóci alsó-Miocén korú Homokköben. Geologica Hungarica, ser Palaeont, 44-46, 260-415.

Lancis, C. y Estévez, A. 1992. Las icnitas de mamíferos del Sur de Alicante (España). Geogaceta, 12, 20-64.
Leonardi, G. 1987. Glossary and manual of tetrapod footprint palaeoichnology. Departamento Nacional do Produçâo Mineral Brasil, 1-118.

López, G., Mayoral, E., Muñoz, A., Pérez, A. y Santamaría, R. 1992. Nuevas icnitas de mamíferos carnívoros en el Mioceno inferior del sector occidental de la Depresión del Ebro (Logroño, La Rioja). Revista de la Sociedad Geológica de España, 5, 39-51.

Panin, N. si Avram, E. 1962. Noi urme de vertebrate în miocenul Sudcarpatilor Romînesti. Studii si cercetari. Geologie, 7, 455-487.

Prats, M. i López, E. 1995. Síntesi de la icnofauna del sinclinal d'Agramunt entre el meridiá d'Agramun i el riu Segre (Prov. de Lleida). Paleontologia $i$ Evolucio, 2829, 247-267.

Renders, E. 1984. The gait of Hipparion sp. from fossil footprints in Laetoli, Tanzania. Nature, 308, 179-181.

Rodríguez Fernández, J . 1982. El Mioceno del sector central de las Cordilleras Béticas. Tesis Universidad Granada, 379, 1-224.

Santamaría, R., López, G., y Casanovas-Cladellas, M.L. 1990. Nuevos yacimientos con icnitas de mamíferos del Oligoceno de los alrededores de Agramunt (Lleida, España). Paleontologia i evolució, 23, 141-152.

Scrivner, P.J. and Bottjer, D.J. 1986. Neogenen avian and mammalian tracks from Death Valley National Monument, California: their context, classification and preservation. Palaeogeography, Palaeoclimatology, Palaeoecology, 57, 285-331.

Serrano, F. 1979. Los foraminíferos planctónicos del Mioceno superior de la cuenca de Ronda y su comparación con los de otras áreas de las Cordilleras Béticas. Serv. Publ. Universidad de Málaga, 272 pp.

Serrano, F. 1992. Biostratigraphic control of Neogene volcanism in the Cabo de Gata (south-east Spain). Geologie en Mijnbouw, 71, 3-14.

Serrano, F., Palmqvist, P., Guerra-Merchán, A. y Romero,A. 1995. Análisis multivariante de las asociaciones de foraminíferos planctónicos de los sedimentos tortonienses de la cuenca de la Atalaya (Cordillera Bética, España). Revista Española de Paleontología, Número homenaje al Dr. G. Colom, 119-128.

Vialov, O.S. 1966. Szledü zsiznedeljatelnoszty organizmov $i$ paleontologicseszkoe znacsénie. Naukova Dumka. Kiev. (Huellas de actividad vital de organismos y su significación paleontológica). Academia Ciencias. Kiev, 1-219. 